\title{
Complicaciones de las derivaciones urinarias heterópicas no continentes: Ureterostomías cutáneas vs conducto ileal
}

\section{Complications of Non-continent Heteropic Urinary Derivations: Skin Ureterostomies vs Ileal Duct}

\author{
Diana Carolina Franco ${ }^{1}$ Rodolfo Varela Ramirez ${ }^{1}$ Byron Lopez de Mesa ${ }^{1}$ Marino Cabrera Fierro ${ }^{1}$ \\ Yesica Yasmín Quiroz Madarriaga ${ }^{1}$ \\ ${ }^{1}$ Departamento de Urología, Instituto Nacional de Cancerología, \\ Bogotá, Colombia \\ Urol Colomb 2019;28:291-295. \\ Address for correspondence Yesica Quiroz Madarriaga, MD, \\ Departamento de Urología, Instituto Nacional de Cancerología, \\ Hospital san José Calle 10 \#18-75, Bogotá, Colombia \\ (e-mail: yeyaquimapa@hotmail.com).
}

\section{Resumen}

Palabras clave

- cáncer urotelial musculoinvasivo

- derivaciones urinarias no continentes heterótopicas

- conducto ileal

- ureterostomias cutáneas
Introducción Y Objetivos Las derivaciones del tracto urinario abarcan una amplia gama de técnicas quirúrgicas, siendo la causa más frecuente para su uso la patología urotelial maligna. De las derivaciones urinarias no continentes heterótopicas las más utilizadas son el conducto ileal y las ureterostomias cutáneas. La elección de la técnica dependerá de la patología de base, las condiciones del paciente y la experiencia del cirujano. El objetivo de este trabajo es determinar las complicaciones tempranas ( $\leq 30$ días) y tardías ( $>30$ días) de derivaciones urinarias heterópicas no continentes (DUHNC) tipo conducto ileal (Cl) vs ureterostomias cutáneas (UC).

Métodos Se realizó un estudio descriptivo retrospectivo de los pacientes llevados a DUHNC tipo Cl o UC, desde enero del 2008 a julio del 2016, en un centro de referencia para patología oncológica. Se evaluó: edad, género, comorbilidades, escala de Karnofsky, patología quirúrgica, sangrado, ASA, tiempo quirúrgico, estancia hospitalaria, complicaciones tempranas y tardías.

Resultados De los 70 pacientes incluidos, 26 con UC y 44 con $\mathrm{Cl}$, prevaleciendo el género masculino en ambos grupos. El promedio de edad fue de 66 y 63 años respectivamente y el indice Karnofsky en todos los pacientes fue superior al $90 \%$. La causa más frecuente fue patología maligna de vejiga. El tiempo quirúrgico fue mayor en los pacientes del $\mathrm{Cl}$, siendo estadísticamente significativo $(p=0.000)$. El sangrado fue similar en ambas técnicas quirúrgicas requiriendo transfusión de hemoderivados el $92,3 \%$ de los pacientes con UC y $88,6 \%$ de los Cl. La estancia hospitalaria no tuvo diferencias. En cuanto a las complicaciones tempranas, la más frecuente en ambos grupos fue el choque hipovolémico $(61 \%$ UC y $58 \% \mathrm{Cl})$. De las complicaciones tardías la sepsis urinaria prevaleció en ambos grupos (34\% y $18 \%$ respectivamente) y la estrechez del estoma y la anastomosis ureteroileal se presentaron en las UC y en los $\mathrm{Cl}$ respectivamente. La mortalidad en el transoperatorio fue del $12,8 \%$. received

February 2, 2018

accepted

July 13,2018
DOI https://doi.org/

$10.1055 / \mathrm{s}-0038-1668513$.

ISSN 0120-789X.

e ISSN 2027-0119.
Copyright $($ C 2019, Sociedad Colombiana License terms de Urología. Publicado por Thieme Revinter Publicações Ltda., Rio de Janeiro, Brazil. Todos los derechos reservados.

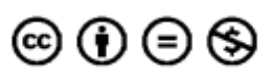




\author{
Abstract \\ Keywords \\ - muscle-invasive \\ urothelial carcinoma \\ - heterotopic \\ incontinent urinary \\ diversion \\ - ileal conduit \\ - cutaneous \\ ureterostomy
}

Conclusiones Las DUHNC como las ureterostomias cutáneas o el conducto ileal son técnicas quirúrgicas con tasas de sangrado, transfusión y estancia hospitalaria similares, pero con una menor proporción de complicaciones tanto tempranas como tardías en los pacientes llevados a conducto ileal.

Introduction and Objectives The derivations of the urinary tract cover a wide range of surgical techniques, being the most frequent cause for its use the malignant urothelial pathology. Of the urinary diversions, the heterotopic continents are the ileal conduit and the cutaneous ureterostomies. The choice of technique will depend on the underlying pathology, the patient's conditions and the experience of the surgeon. The aim of this study is to determine the early complications ( $\leq 30$ days) and late complications (> 30 days) of noncontinental heteropic urinary diversions (NHUD) type ileal conduit (IC) vs cutaneous ureterostomies (CU).

Methods A retrospective descriptive study was conducted of patients referred to NHUD type $\mathrm{Cl}$ or CU, from January 2008 to July 2016, in a referral center for oncological pathology. We evaluated: age, gender, comorbidities, Karnofsky scale, surgical pathology, bleeding, ASA, surgical time, hospital stay, early and late complications.

Results Of the 70 patients included, 26 with CU and 44 with IC, the male gender prevailing in both groups. The average age was 66 and 63 years respectively and the Karnofsky index in all patients was over $90 \%$. The most frequent cause was malignant pathology of the bladder. Surgical time was higher in IC patients, being statistically significant $(p=0.000)$. Bleeding was similar in both surgical techniques requiring transfusion of blood products $92.3 \%$ of patients with CU and $88.6 \%$ of IC. The hospital stay did not differ. Regarding the early complications, the most frequent in both groups was hypovolemic shock $(61 \% \mathrm{CU}$ and $58 \% \mathrm{Cl})$. Of the late complications, urinary sepsis prevailed in both groups (34\% and 18\% respectively) and the narrowing of the stoma and the ureteroileal anastomosis were present in the CU and in the IC, respectively. The mortality in the transoperative period was $12.8 \%$.

Conclusions NHUD such as cutaneous ureterostomies or ileal conduit are surgical techniques with similar rates of bleeding, transfusion and hospital stay, but with a lower proportion of complications both early and late in patients taken to the ileal conduit.

\section{Introducción}

La cistectomía radical es el gold estándar para el manejo de cáncer de vejiga musculoinvasivo, con tasas de supervivencia global a 5 años que oscilan entre el $48 \%$ al $70 \%$. No obstante, dicha técnica también se ha brindado para manejar secuelas de la radioterapia secundarios al control de patologías pélvicas como son los casos de cistitis actínica o fistulas de difícil manejo cuya resolución contribuye a una mejor calidad de vida de los pacientes.

Desde 1859 Simon y col., describieron derivaciones urinarias que hasta la década de los 50 se pudieron llevar a cabo de manera exitosa por Bricker, siendo el CIy las UC las más frecuentemente utilizadas, pero no las únicas descritas, cada una con ventajas y desventajas, complicaciones y desenlaces, por lo que la elección de la técnica dependerá de la patología de base, las condiciones del paciente y la experiencia del cirujano. El objetivo de este trabajo es determinar las complicaciones tempranas ( $\leq 30$ días) y tardías (>30 días) de derivaciones urinarias heterópicas no continentes (DUHNC) tipo conducto ileal (CI) vs ureterostomias cutáneas (UC).

\section{Métodos}

Se realizó un estudio descriptivo retrospectivo de los pacientes llevados a DUHNC tipo CI o UC, desde enero del 2008 a julio del 2016, en un centro de referencia para patología oncológica, tanto por diagnostico benigno como maligno, identificando 129 pacientes llevados a cistectomía radical más derivación urinaria, de los cuales 84 se trataban de CI o UC. Se excluyeron aquellos pacientes en quien se llevó a cabo intervenciones adicionales en el mismo tiempo quirúrgico para finalmente incluir 70 pacientes en quienes se evalúo: edad, género, comorbilidades, escala de Karnofsky, patología quirúrgica, sangrado, ASA, tiempo quirúrgico, estancia hospitalaria, complicaciones tempranas y tardías.

Todas las piezas quirúrgicas se evaluaron en el departamento de patología de la institución. Las complicaciones tempranas se 
definieron como aquellas que se presentaron en el primer mes POP y las tardías al cumplir dicho lapso.

Los datos se recolectaron mediante una base de datos creadas en excel y se procesaron mediante STATA 13.0, en donde se analizaron frecuencias absolutas, porcentajes, tasas, desviaciones estándar y rangos intercuartilicos. Y se utilizó el índice de Fisher como medida de asociación de variables cualitativas con punto de corte estadísticamente significativo en 0,05 .

\section{Resultados}

De los 70 pacientes incluidos, 26 tuvieron UC y $44 \mathrm{CI}$, prevaleciendo el género masculino en ambos grupos. El promedio de edad fue de 65 años y el indice Karnofsky en la mayoría de los pacientes fue superior al $90 \%$. Las características generales de la población se describen en la - Tabla 1.

El tiempo quirúrgico fue mayor en los pacientes del $\mathrm{CI}$, siendo estadísticamente significativo $(p=0.000)$. El sangrado fue similar en ambas técnicas quirúrgicas requiriendo transfusión de hemoderivados el $92,3 \%$ de los pacientes con UCy 88,6\% de los CI. -Tabla 2. La técnica quirúrgica empleada para la anastomosis ureterointestinal en $\mathrm{Cl}$ fue Wallace en el 36,3\% y

Tabla 1 Caracterización población

\begin{tabular}{|c|c|c|}
\hline & POBLACIÓN (n:70) & $\%$ \\
\hline Edad, Riq & $65(60-69)$ & \\
\hline \multicolumn{3}{|l|}{ Sexo } \\
\hline Femenino & 20 & 28,5 \\
\hline Masculino & 50 & 71,4 \\
\hline \multicolumn{3}{|l|}{ Comorbilidades } \\
\hline No & 35 & 50 \\
\hline Hta & 30 & 42,8 \\
\hline $\mathrm{Dm}$ & 8 & 11,4 \\
\hline Otros & 9 & 12,9 \\
\hline \multicolumn{3}{|l|}{ Escala Karnofsky } \\
\hline 100 & 31 & 44,2 \\
\hline 90 & 35 & 50 \\
\hline 80 & 4 & 5,7 \\
\hline \multicolumn{3}{|l|}{ Derivación Urinaria } \\
\hline Ureterostomía & 26 & 37,1 \\
\hline Conducto lleal & 44 & 62,8 \\
\hline \multicolumn{3}{|l|}{ Patología } \\
\hline Urotelial Alto Grado & 54 & 77,1 \\
\hline Urotelial Bajo Grado & 3 & 4,2 \\
\hline Adenocarcinoma & 1 & 1,4 \\
\hline Escamocelular & 5 & 7,1 \\
\hline Negativo Para Malignidad & 4 & 5,6 \\
\hline Leiomiosarcoma & 3 & 4,2 \\
\hline \multicolumn{3}{|c|}{ Intervención Quirúrgica por Patología no Oncológica } \\
\hline & 1 & 1,4 \\
\hline
\end{tabular}

Bricker en 63,6\%. En cuanto a las UC 73\% fueron bilaterales y en la mayoría de los casos se realizó por inestabilidad hemodinámica durante el transoperatorio (57,6\%).

La estancia hospitalaria no tuvo diferencias. En cuanto a las complicaciones tempranas la más frecuente en ambos grupos fue el choque hipovolémico (61\% UC y 58\% CI). De las complicaciones tardías la sepsis urinaria prevaleció en ambos grupos (34\% y 18\% respectivamente), la estrechez del estoma se presentó en las UC y en el grupo de CI la estrechez de la anastomosis ureteroilieal. Las complicaciones se especifican en la - Fig. 1. La mortalidad en el transoperatorio fue del $11,5 \%$ y $13,6 \%$ respectivamente.

\section{Discusión}

La primera publicación en donde se usó segmento intestinal como derivación urinaria fue en 1851 por Simon para corregir casos de extrofia vesical, sin embargo, dado la alta tasa de mortalidad se consideró limitar su uso. ${ }^{1}$ Desde entonces, diversas técnicas fueron ajustadas con el fin de disminuir complicaciones tales como fugas anastomóticas, compresiones ureterales, infecciones, necrosis o perforación intestinal. En 1950 Bricker popularizó el conducto ileal al seleccionar en segmento intestinal como reservorio, disminuyendo las complicaciones e instaurando la derivación urinaria no continente como la primera elección posterior a una cistectomía. $^{2}$ No obstante, al intentar disminuir la morbilidad ocasionada por la manipulación del segmento intestinal y reducir el tiempo operatorio, Obrant en 1957, describió la trasureteroureterostomia con ureterostomia cutánea la cual fue popularizada por Hodges en $1980^{3}$.

Las complicaciones en las reconstrucciones urinarias junto con las cistectomías son altas ya que oscilan desde un $62 \%$ a un $78 \%$, con tasas de mortalidad que van del 0,3 al $9,5 \%,{ }^{4-6}$ siendo la mayoría de las complicaciones menores (Clavidien 1 o 2) pero que podrían aparecer hasta 20 años después por lo que requieren seguimiento de por vida ${ }^{7}$.. Kuroda y col analizaron 237 pacientes llevados a cistectomía radical con recontrucción urinaria con CI o UC en Osaka, reportando complicaciones tempranas en el 50\% y 37\% de los pacientes llevados a CI y UC respectivamente, con complicaciones tardías en el $45 \%$ y el $47 \%{ }^{3}$ En nuestra población, las complicaciones tempranas alcanzaron el 66 y el $73 \%$ en CI y UC respectivamente, disminuyendo después del mes a 45 y 61\%, lo cual se encuentra acorde con lo reportado en la literatura, cuando se reportan las complicaciones globales de las DUHNC, pero cuando se comparan por separado las técnicas quirúrgicas, las UC siempre informan menores tasas de complicación a diferencia de nuestra población, en donde fueron el grupo que más morbilidad tuvo en el POP temprano y tardío. Eso se debe a que la UC se realizó por complicaciones en el intraoperatorio de los pacientes que inicialmente estaban programados para otra derivación urinaria. En cuanto a la mortalidad, en este estudio alcanzo el 12,8\%, siendo mucho más alto que lo reportado en las cohortes más grandes de derivaciones urinarias, probablemente por tratarse de una población menor. 
Tabla 2 Seguimiento Trans Y Perioperatorio Derivación Urinaria Heterotópica No Continente

\begin{tabular}{|l|l|l|l|l|l|}
\hline & Ureterostomía (n:26) & $\%$ & Conducto lleal (n:44) & $\%$ & \\
\hline Edad, mediana (riq) & $66,5(63-71)$ & & $64,5(59-68)$ & & $p=0,137$ \\
\hline ASA & & & & & $p=0,919$ \\
\hline I & - & - & 1 & 2,2 & \\
\hline II & 9 & 34,6 & 13 & 29,5 & \\
\hline III & 17 & 65,3 & 29 & 65,9 & \\
\hline IV & - & - & 1 & 2,2 & \\
\hline Sangrado (cc), mediana (riq) & $1200(600-2000)$ & & $1300(700-1500)$ & & $p=0,840$ \\
\hline Tiempo quirúrgico (min), mediana (riq) & $297(250-360)$ & & $427(377-515)$ & & $p=0,000$ \\
\hline Transfusión hemoderivados & 24 & 92.3 & 39 & 88,6 & $p=1,000$ \\
\hline Uci Pop, Pte & 21 & 80.7 & 43 & 97,7 & $p=0,024$ \\
\hline Estancia hospitalaria (día), mediana (riq) & $14(10-20)$ & & $13(11-17)$ & & $p=0,854$ \\
\hline Mortalidad trans-perioperatorio & 3 & 11.5 & 6 & 13,6 & \\
\hline
\end{tabular}

Los primeros informes donde documentaron las complicaciones propias del conducto ileal fueron descritos por Nurmi y col en 1988, donde evaluaron 144 pacientes quienes presentaron complicaciones tempranas en el $33 \%$ de los casos, incluyendo: infecciones de la herida quirúrgica, fuga ureteroileal, obstrucción intestinal, fistulas o ITU; y tardías de $28 \%$ relacionadas al estoma y al deterioro de la función renal, ${ }^{8}$ al igual que Neal en 1985 quien informo tasas de IRC hasta del $16 \%$ relacionadas con ITU a repetición secundarias a reflujos ureterales no controlados. ${ }^{2}$ De igual manera Pekarovic en la década de los 80 informo complicaciones tardías del conducto ileal confinadas al estoma tales como estenosis, dermatitis, prolapsos, retracciones o hernias paraestomales los cuales oscilaban del $15 \%$ al $43 \% .^{8}$ A la fecha, las tasas globales de complicaciones asociadas al conducto ileal corresponden a pielonefritis en el 5\%-23\%, obstrucción ureteral 2\%-22\%, urolitiasis 3\%-16\% y complicaciones del estoma del $2 \%-62 \%{ }^{9}$ En nuestra cohorte, y dentro de las complicaciones tempranas, se destacan shock hipovolémico 56\%, alteraciones relacionadas con la manipulación intestinal en $10,6 \%$, seguidas de hipoperfusión del estoma en el $9 \%$ e ITU en $4,6 \%$, lo cual es menor a lo reportado en la literatura, a excepción del sangrado intraoperatorio el cual es menor en las grandes series con promedio de $490 \mathrm{cc}$ vs los $1300 \mathrm{cc}$ que en el grupo de CI, lo cual se explica por la complejidad de los pacientes llevados a cistectomía radical. En cuanto a las complicaciones tardías de ese grupo, se presentaron en orden de frecuencia: estrecheces ureteroiliales $24,8 \%$, seguida de sepsis urinaria

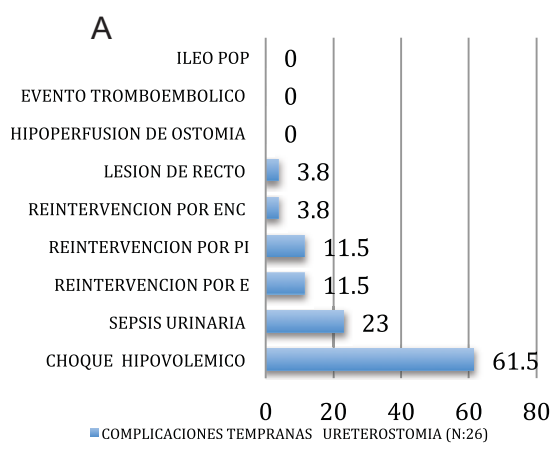

B

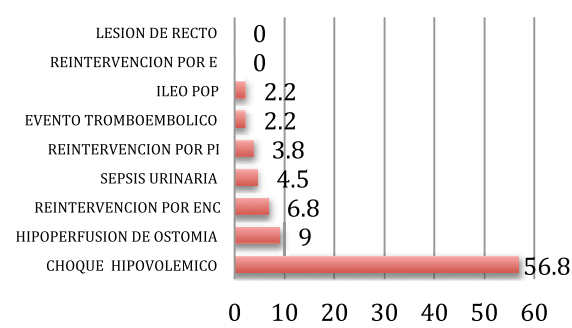

- COMPLICACIONES TEMPRANAS CONDUCTO ILEAL (N:44)

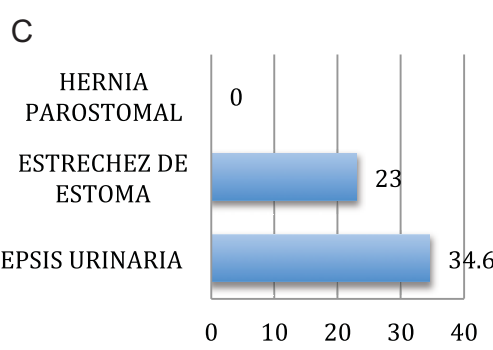

= COMPLICACIONES TARDIAS URETEROSTOMIA (N:26)

D

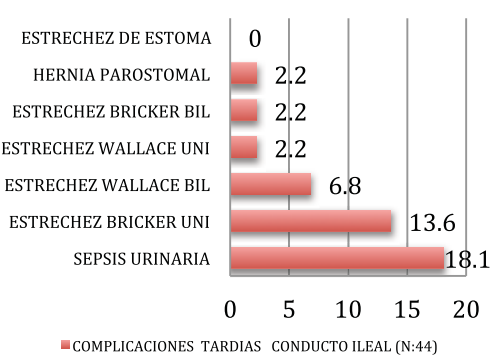

Fig. 1 (A) Complicaciones tempranas de UC, (B) Complicaciones tempranas de $\mathrm{Cl}$, (C) complicaciones tardías de UC, (D) complicaciones tardías de Cl. +ENC: evisceración no contenida; +PI: perforación intestinal; +E: empaquetamiento 
$18,1 \%$ y hernias paraestomales en $2,2 \%$, siendo menor la proporción de hernias por el anclaje del estoma al músculo que se realiza de manera rutinaria en nuestra institución.

Feminella y col., en 1971 describieron los primeros informes donde evidenciaron una alta incidencia de estenosis del estoma, abscesos y necrosis ureteral alcanzando tasas del 60 al 70\% en pacientes llevados a ureterostomia cutánea, lo cual ha disminuido con el uso de stent ureteral para la permeabilización del trayecto. ${ }^{3}$ De los 26 pacientes llevados en nuestra población a UC, el 73,1\% presentó complicaciones en el primer mes como: shock hipovolémico 61,5\%, ITU 23\%, y complicaciones intestinales $15,3 \%$, lo cual no se describe con frecuencia en la literatura ya que se enfocan más en las complicaciones tardías las cuales en nuestra cohorte fueron en orden de frecuencia sepsis urinaria en 34,6\% y estrechez del estoma en el $23 \%$, el cual es menor al reportado en la literatura por el uso habitual de catéteres JJ durante la realización del estoma. En cuanto a la sepsis se presenta de manera más frecuente en esa población por la fácil colonización del tracto urinario superior al no existir reservorio.

La etiología de las estenosis se ha relacionado con la isquemia que presenta el uréter a nivel distal, incrementado su incidencia por la movilización excesiva del uréter junto con la realización del túnel mesentérico estrecho, alterando el aporte vascular de dicho segmento, ${ }^{4}$ siendo su corrección abierta la mejor opción para dicha complicación dado que el manejo endoscópico inicial podría de hecho disminuir el éxito de la reparación de esta estenosis. ${ }^{10,11}$

Deliveliotis y col., en el 2005, comparó las dos técnicas quirúrgicas encontrando que el tiempo quirúrgico, el sangrado y la necesidad de transfusión fueron menores en el grupo de ureterostomias cutáneas, reportando esa diferencia estadísticamente significativa. ${ }^{8,12}$ En nuestro estudio, el tiempo quirúrgico fue de 297 vs 427 minutos $(p=0,00)$ y la necesidad de UCI POP fue de 80,7 vs $97,7 \%$ ( $p=0,024)$ para UC y CI respectivamente, ya que la mayoría de los pacientes en ambos grupos fueron ASA III, lo que favorecía la tasa de requerimiento de UCI POP. En cuanto al sangrado, la tasa de transfusión (UC 92,3\% y CI 88,6\%, $p=1,00$ ) y la estancia hospitalaria no fueron estadísticamente significativos.

\section{Conclusión}

Con la depuración de la técnica quirúrgica y la mejoría en los cuidados POP han disminuido las complicaciones, permitiendo al personal médico identificar factores predictivos $\mathrm{y}$ pronósticos para decidir la derivación de elección ofreciendo menor potencial de complicaciones y mejor calidad de vida.
Las DUHNC como las ureterostomías cutáneas o el conducto ileal son técnicas quirúrgicas con tasas de sangrado, transfusión y estancia hospitalaria similares, pero con una menor proporción de complicaciones tanto tempranas como tardías en los pacientes llevados a conducto ileal.

\section{Conflicto de intereses}

Los autores declaran no tener ningún conflicto de intereses.

\section{Referencias}

1 Williams $\mathrm{O}$, Vereb MJ, Libertino JA. Noncontinent urinary diversion. Urol Clin North Am 1997;24(04):735-744

2 Madersbacher S, Schmidt J, Eberle JM, et al. Long-term outcome of ileal conduit diversion. J Urol 2003;169(03):985-990

3 Kilciler M, Bedir S, Erdemir F, Zeybek N, Erten K, Ozgok Y. Comparison of ileal conduit and transureteroureterostomy with ureterocutaneostomy urinary diversion. Urol Int 2006;77(03): 245-250

4 Gin GE, Ruel NH, Parihar JS, et al. Ureteroenteric anastomotic revision as initial management of stricture after urinary diversion. Int J Urol 2017;24(05):390-395

$5 \mathrm{Kim} \mathrm{SH}$, Yu A, Jung JH, Lee YJ, Lee ES. Incidence and risk factors of 30-day early and 90-day late morbidity and mortality of radical cystectomy during a 13-year follow-up: a comparative propensity-score matched analysis of complications between neobladder and ileal conduit. Jpn J Clin Oncol 2014;44(07): 677-685

6 De Nunzio C, Cindolo L, Leonardo C, et al. Analysis of radical cystectomy and urinary diversion complications with the Clavien classification system in an Italian real life cohort. Eur J Surg Oncol 2013;39(07):792-798

7 Hautmann RE, Abol-Enein H, Davidsson T, et al; International Consultation on Urologic Disease-European Association of Urology Consultation on Bladder Cancer 2012. ICUD-EAU International Consultation on Bladder Cancer 2012: Urinary diversion. Eur Urol 2013;63(01):67-80

8 Nurmi M, Puntala P, Alanen A. Evaluation of 144 cases of ileal conduits in adults. Eur Urol 1988;15(1-2):89-93

9 Lee RK, Abol-Enein H, Artibani W, et al. Urinary diversion after radical cystectomy for bladder cancer: options, patient selection, and outcomes. BJU Int 2014;113(01):11-23

10 Schöndorf D, Meierhans-Ruf S, Kiss B, et al. Ureteroileal strictures after urinary diversion with an ileal segment-is there a place for endourological treatment at all? J Urol 2013; 190(02):585-590

11 Wolf JS Jr, Elashry OM, Clayman RV. Long-term results of endoureterotomy for benign ureteral and ureteroenteric strictures. J Urol 1997;158(3 Pt 1):759-764

12 Deliveliotis C, Papatsoris A, Chrisofos M, Dellis A, Liakouras C, Skolarikos A. Urinary diversion in high-risk elderly patients: modified cutaneous ureterostomy or ileal conduit? Urology 2005;66(02):299-304 